\section{A spotlight on microneurotrophins: the future of amyotrophic lateral sclerosis treatment?}

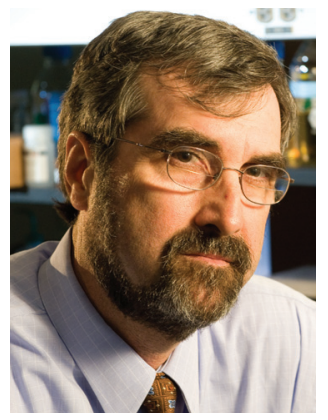

James P Bennett Jr* speaks to Daphne Boulicault, Commissioning Editor: James P Bennett, Jr is a native of St Petersburg, FL, USA. He received a BS in chemistry (with Honors) from the University of Florida (1970), then attended Johns Hopkins University School of Medicine for his MD (1974) and PhD (pharmacology, 1977) degrees. He worked in the laboratory of Dr Solomon Snyder, MD, for 6 years while in medical and graduate schools and trained in neuropharmocology. He then trained clinically for 2 years in internal medicine (1978-1980), followed by Neurology residency at the University of Virginia (UVA; 1980-1983) where he was Chief Resident (1982-1983). He became a faculty member in the UVA Departments of Neurology, Psychiatry and Pharmacology (1983-2009) and held the Ebbert Chair in Medical Science. In 2009, he moved to Virginia Commonwealth University, where he is the Bemiss Professor, was Chair of Neurology (2009-2013) and is founding Director of the Parkinson's Disease and Movement Disorder Center (2009-2015). He has held multiple NIH grants and directed the NIH-funded Udall Parkinson's Center at UVA. He investigates mitochondrial dysfunction in adult neurodegenerative diseases. He holds multiple patents related to experimental therapies of degenerative disorders.

\section{Q Could you give us a brief overview of your career to date?}

I was originally a chemistry major who was persuaded to attend medical school by a friend of the family and neurosurgeon, named John Thompson, who worked in private practice. He helped me get into medical school at John Hopkins, where I spent the summers working at a pharmacology research laboratory and decided that this was where my interests lay.

At the time, there was no MD-PhD program offered at my medical school, but students were allowed to complete their last year as an elective. This allowed me to merge medical school and graduate school. I graduated from medical school with my class, then finished my $\mathrm{PhD}$ in neuropharmacology.

After completing my PhD, I decided to pursue clinical training with 2 years of internal medicine and 3 years of neurology. Following this, I joined the faculty at the University of Virginia as an Assistant Professor in neurology with the aim of becoming a physician scientist. Early on I became interested in Parkinson's disease, in which I am to this day primarily focused on, and began a laboratory and clinical research program in the field.

In sum, I have devoted my career to understanding the neurobiology of neurodegenerative disease, over time expanding from Parkinson's disease into Alzheimer's and amyotrophic lateral sclerosis (ALS). I have further worked toward the development

*Neurodegeneration Therapeutics, Inc., 3050A Berkmar Drive, Charlottesville VA 22902, USA; jpb8u@me.com

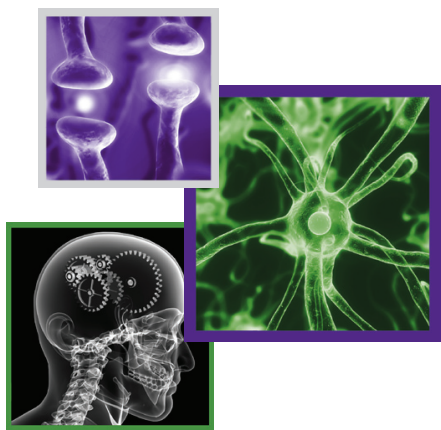

Future $\because \cdots$ Medicine 
of experimental therapies based on our findings and carried out clinical research into these myriad of diseases. Ultimately, I have developed into a translational physician scientist, though 'translational' was not a recognized term when I first began my career.

The evolution of my career is marked by the 10-year span of my Udall Center for Parkinson's disease and the establishment of my Parkinson's and Movement Disorders Center at Virginia Commonwealth University (VCU) in 2009. The VCU Parkinson's and Movement Disorders Center is in many ways a representation of my career, as an experimental, multidisciplinary clini$\mathrm{cal}$ and translational research operation. The last 6 years of my career have been concerned with the formation of this center. In 2015 I left VCU to form a non-profit company focused on using stem cell technology to "personalize" pharmacological approaches to neurodegenerative disease therapies.

Q What first inspired you to work in the field of neurodegenerative disease?

John Thompson, who I mentioned previously, would have me accompany him on rounds and to the operating room when I was on school breaks, with the aim of convincing me to study brain chemistry. During one of my visits to his office, he told me about a patient of his who had Parkinson's disease, at a time when L-DOPA had just been introduced in the USA. During an appointment, some months into the development of her L-DOPA treatment regimen, she began to cry. When John asked what was wrong, she replied that they were tears of joy because for the first time in 10 years she was able to brush her own teeth. As a chemistry major, I found this story incredibly striking.

It was then that I decided to explore Parkinson's disease. On returning to medical school, I joined up with a neuropharmacological laboratory and, as they say, I took to it like a duck to water - it was a perfect fit!

Q Are there any other colleagues that have particularly influenced you \& your career?

Absolutely, another role model of mine was Dr Solomon Snyder. Although trained in psychiatry, he ran a molecular pharmacology research laboratory. He was interested in showing how understanding of the brain leads to better treatments. His was the first laboratory I worked at during the summer of medical school and, later, throughout my $\mathrm{PhD}$.
'Sol' was a major influence both as a $\mathrm{PhD}$ mentor and as a friend. He taught me how to run a productive laboratory, to sensitively handle the personal issues that arise among colleagues and to be an effective mentor. I have gone on to become a successful mentor myself - I hope - and thoroughly enjoy the role.

Sol's laboratory was populated with neurologists who enlightened me to the fact that one could be a physician while continuing one's study of the brain. These were incredibly enjoyable years for me and I believe the influence of scientists from a range of different, yet related, fields had a large positive impact on me.

\section{Q What would you describe as the biggest achievement of your career?}

I always hesitate in answering this question but I would say that my biggest achievement scientifically is my participation in the study of mitochondrial dysfunction in neurodegenerative diseases. This is mated with my good fortune in having worked with wonderful people and talented scientists, some of whom I continue to collaborate with to this day. Third, my role as a mentor has been profoundly satisfying and I am about to graduate my 11 th $\mathrm{PhD}$ student!

Q As one of the lead investigators for the ALS MicroNeurotrophin Research Consortium, can you first provide us with some background on microneurotrophins \& their relevance to ALS?

Microneurotrophin is a class descriptor referring to a series of small-molecule analogues that are derived from the structure of a naturally occurring steroid precursor, known as dehydroepiandrosterone (DHEA). The DHEA compound has been transformed into a series of analogues by a Greek pharmacologist named Achilleas Gravanis from the University of Crete. These analogues potently and selectively mimic the actions of large neurotrophin proteins at their tyrosine kinase (Trk) receptors.

Neurotrophin proteins are large, well-known protein molecules with a number of beneficial effects on neurons. Essentially, they help neurons grow, differentiate and survive stress. However, for therapeutic use, they must be pumped directly into the brain or they break down and do not cross the blood-brain barrier.

What Achilleas has done is to create smallmolecule drugs (which can be administered as pills, for example) that are absorbed into the brain, are nontoxic and mimic the actions of 
neurotrophins. As a consortium, we decided that they looked extremely promising as therapies for neurodegenerative disease.

\section{Q How was the ALS MicroNeurotrophin} Research Consortium founded, \& who are the key players involved?

I was approached by ALS Worldwide to be a part of the Consortium to aid the development of one or more of these analogues for submission to the US FDA and the EMA, in order to obtain Investigational New Drug Applications (INDs) for their experimental use in humans. The routine short-term animal toxicity that the FDA and the EMA require represents investment of around US $\$ 2$ million per drug, so it is important to pick your candidates carefully.

I was selected as a lead on the team for a number of reasons: first, I have a physician-sponsored IND from the FDA for another ALS drug I developed; second, I have as much experience with ALS as with other degenerative diseases I have studied; and third, I believe I am a good communicator, which is useful for explaining our work to the interested public and to investors.

After I joined, we, as a consortium, held two meetings: one in Boston and one in Sheffield, UK. We came away with about half a dozen investigators from Harvard, Sheffield, Pennsylvania State, my laboratory at VCU and Achilleas Gravanis' company that owns the intellectual property for these drugs.

As a group, we are very open with one another and our goal is to gather enough preclinical information to secure INDs from the FDA and the EMA. Dame Professor Pamela Shaw from Sheffield University will be responsible for liaising with the EMA, while I will take the lead with the FDA.

In essence, what we have formed is a 'homegrown' drug development program, but instead of the more classic single-protein target, we are aiming to be able to affect multiple processes within vulnerable neurons.

Q Can you describe some of the work of the ALS MicroNeurotrophin Research Consortium so far?

The goal of the Consortium is to increase our knowledge generally, but specifically to select one or two analogues for development in humans. Therefore, we bear in mind how the FDA and the EMA, who are fairly conservative, work. These regulatory bodies look toward effects in animal models in addition to other cell biology (even though animal model results typically translate rather poorly in humans).

Currently, we are considering four microneurotrophin analogues and evaluating which have the best chances of helping people with either ALS or other conditions. We have programs involving the application of our drugs in zebrafish and transgenic mouse models of familial ALS. In fact, we already have preliminary results that suggest one of the analogues has a neuroprotective effect in one of the ALS mouse models. We also know that they can be given to animals for weeks and months without any toxicity, so they are probably nontoxic. There are, of course, other types of data that will be taken into account and we are working on these too.

\section{Q What work is your laboratory conducting} to support the Consortium?

Primarily, my lab is creating human motor neurons from various stem cell types (some of which we also create). We expose these human motor neurons to the microneurotrophin analogues and analyze which genes get activated or repressed using RNASeq. We then compare these cells with ALS and control spinal cord samples to discover which analogues 'correct' the abnormalities found in ALS spinal cords.

Q What will be the priorities for the next 5 years, both for your lab \& the Consortium as whole?

The main priority is to get physician-sponsored INDs and to introduce one or more of these molecules into humans with ALS. Of course, to begin with we would be administering these molecules in very small numbers of humans while monitoring their toxicity. Then, we will gradually increase the dose as the toxicity profile hopefully stays manageable.

As I often say, the highway of neurodegenerative drug therapy is littered with the carcasses of failed drug trials. This has been the case for all the major diseases, so ironically there is almost an expectation of failure. Nonetheless, it remains of the utmost importance to try, and that is what our consortium is doing.

Within this 5-year horizon, we need to develop approaches to sort people into molecular phenotypes, as has been done within breast cancer. We need to become smart, like our cancer biology colleagues, in order to create specific and individualized MicroNeurotrophin therapies for 
patients. I fully expect for there to be differences, at the molecular level, among ALS patients.

The hope, of course, is that these drugs will have the potential for application in other diseases. Once we have made headway within ALS, it will be more practical to amend the IND for application in other diseases, particularly as INDs are issued for a molecule rather than a specific condition. It makes sense to begin the process in ALS, an untreatable, rapidly progressive and fatal disease for which there is a crying need for insights and new approaches.

Q You are also the Director of the VCU Parkinson's Disease Center, what recent or forthcoming developments have you excited within this role?

The approach for the Parkinson's Disease Center is to produce translational, hybrid research through laboratory and clinical efforts, in order to push knowledge from 'bench to bedside'. There are several imminent programs that I am particularly proud of.

One project is being run by our neuropsychology group, with Dr Sarah Lageman as the lead. Cognitive impairment is a serious problem that is not spoken about enough; we have found that $50-60 \%$ of patients that walk through the door of a center like ours have some level of mild cognitive impairment, while $20-25 \%$ are overtly demented. This represents a huge problem within the Parkinson's population and was reported in Brain (2010) to be the major source of disability. Sarah's program uses computer memory-training in Parkinson's patients with cognitive impairment. Her preliminary data look extremely promising in terms of improving memory function with long-term benefits.

Dr Katherine Holloway, our functional neurosurgeon who works on deep brain stimulation and electrode placement, is in the process of getting an investigational device exemption (IDE) from the FDA. She hopes to be able to implant electrodes into the nucleus basalis of Meynert (NBM). The NBM is an area that supplies acetylcholine to the forebrain and is particularly involved in Parkinson's dementia and to a slightly lesser extent in Alzheimer's disease. Katherine aims to use the electrodes to stimulate the residual cholinergic neurons in order to improve memory function.

The third project involves low-dose brain radiation to treat amyloidosis in the brain. Some of the studies using animal models of Alzheimer's disease suggest that amyloidosis accounts for some of the cognitive impairment and memory dysfunction. Leland Rogers, one of my radiation oncology colleagues, is developing a protocol in which individuals with early-tomoderate Alzheimer's disease receive a very low dose of brain irradiation. The hope is that the plaques will recede as they do in animal models.

In addition to these, I am extremely proud of the continued work my laboratory group is producing in relation to mitochondrial research. We have also established a brain bank which is coming along nicely.

Clinically speaking, we have established multidisciplinary evaluation and treatment of our Parkinson's patients. This has had an enormously positive effect on our patients and the center is now more popular than ever, having seen over 1000 Parkinson's disease patients in the last 3 years!

\section{Q How do you see your field progressing in the next 5-10 years?}

First, the scientific community depends on research support. At the moment, in the USA at least, the federal government seems to be doing their utmost to eliminate support for any type of basic science, the NIH is withering on the vine and laboratories are closing. Additionally, donors have not seemed to have gotten the message that they need to step up to the plate. If this issue of support does not change, then all the valuable work will vanish; this is an incredibly draconian and bleak outlook, but it is the reality as I see it.

The positive side is that we now have some amazing molecular genetic tools as a result of public investment in science - driven mainly by the cancer biologists who consistently take the lead here - that we could only have dreamed of 5 years ago. It is truly amazing to think that the Human Genome Project is 10 years old and the cost of sequencing a genome has fallen from billions of dollars to around US\$2000. We are on the verge of applying these tools to define the molecular uniqueness of each of us, including those with degenerative disease, and to therefore develop personalized therapies. All we need is the public support to do the experiments and to generate the needed data.

In 5-10 years, I would like for a lot of these degenerative disease to be put to bed in a similar way to HIV, in that the diseases still exist but patients are able to lead an almost normal lifespan of good quality. With continued public support, I believe this goal is attainable. 


\section{Disclaimer}

The opinions expressed in this interview are those of the interviewee and do not necessarily reflect the views of Future Medicine Ltd.

\section{Financial \& competing interests disclosure}

$J P$ Bennett is the inventor for issued US and EU patents involving the use of $R(+)$ pramipexole (PPX) for degenerative diseases. $R(+)$ PPX is not specifically discussed in this article. The authors have no other relevant affiliations or financial involvement with any organization or entity with a financial interest in or financial conflict with the subject matter or materials discussed in the manuscript apart from those disclosed.

No writing assistance was utilized in the production of this manuscript. 